\begin{tabular}{lllll}
\hline \multicolumn{6}{l}{ Abstract PWE- 182 Table 1 } & & \\
\hline Year & 30 Day Mortality & & 1 Year Mortality \\
\hline 2005 & 11 & $21.6 \%$ & 31 & $60.8 \%$ \\
2006 & 4 & $13.3 \%$ & 12 & $40.0 \%$ \\
2007 & 4 & $13.3 \%$ & 13 & $43.3 \%$ \\
2008 & 0 & $0.0 \%$ & 7 & $36.8 \%$ \\
2009 & 4 & $11.8 \%$ & 14 & $41.2 \%$ \\
2010 & 6 & $15.4 \%$ & 18 & $46.2 \%$ \\
2011 & 1 & $3.8 \%$ & 5 & $19.2 \%$ \\
2012 & 2 & $6.7 \%$ & 6 & $20.0 \%$ \\
Mean & 4 & $10.7 \%$ & 13.25 & $38.4 \%$ \\
\hline
\end{tabular}

effective. 1 year mortality in MND patients is high and emphasises the importance of careful patient selection.

We recommend that other institutions with a high 30 day mortality following PEG adopt the changes in practise outlined above. Ongoing audit of our practise is required.

Disclosure of Interest None Declared.

\section{PWE-183 DOES INSERTION OF A GASTROSTOMY CONFER ANY QUALITY OF LIFE BENEFIT TO EITHER PATIENTS OR THEIR CARERS?}

doi:10.1136/gutjnl-2013-304907.471

1." M Kurien, 'M E McAlindon, ' $\mathrm{J}$ Grant, ' $\mathrm{E} F \mathrm{~F}$ Wong, ${ }^{1} \mathrm{~A}$ J Johnston, ${ }^{2} \mathrm{~B}$ Hoeroldt, ${ }^{3} \mathrm{~K} \mathrm{~L}$ Dear, 'D S Sanders. 'Department of Gastroenterology, Royal Hallamshire Hospital, Sheffield; ' ${ }^{2}$ Department of Gastroenterology, Rotherham Hospital, Rotherham; ${ }^{3}$ Department of Gastroenterology, Chesterfield Royal Hospital, Chesterfield, UK

Introduction Gastrostomy feeding is an effective means of providing enteral nutrition to patients who have functionally normal gastrointestinal tracts but who cannot meet their nutritional requirements because of an inadequate oral intake. Whilst improvements in outcome measures such as nutritional status and mortality have been demonstrated in certain patients undergoing gastrostomy insertion, there remains a paucity of work evaluating another important health outcome measure, which is health related quality of life (HRQoL). Furthermore no previous study has looked at the impact on carers' HRQoL when considering all referral indications for gastrostomy. This prospective, multicenter study evaluates HRQoL in both gastrostomy patients and their carers, with comparisons made with a population control group.

Methods 61 patients (mean age 68 years) and 58 carers (mean age 65 years) were prospectively recruited from 4 hospitals in South Yorkshire between Feb-Dec 2012. All individuals had HRQoL evaluated prior to gastrostomy insertion, with repeated measurements undertaken at 3 months. Assessment was undertaken using EQ-5D, a validated assessment tool and preferential measure used by NICE, producing scores between 0 for dead and 1 for perfect health. Findings were then compared with a separate cohort of population controls ( $n=419)$, to determine if differences existed in HRQoL. Non-parametric statistical analysis was undertaken using a Wilcoxon Rank test to compare longitudinal paired EQ-5D scores, and a Mann-Whitney test to compare EQ-5D scores between groups, with $p$ values $<0.05$ considered significant.

Results 61 gastrostomy patients have been assessed to date. Of these, 3 died prior the 3 month reassessment post insertion. No significant change was shown in mean EQ-5D scores in either the gastrostomy patients $(0.74$ versus $0.73, p=0.11)$ or their carers ( 0.96 versus $0.97, p=0.30$ ) at 3 months following gastrostomy insertion. When compared to population controls, carers had comparable scores to the population controls unlike the gastrostomy patients who had significantly lower mean EQ-5D scores (0.73 versus $0.94, \mathrm{p}<0.0001)$.
Conclusion This study demonstrates that HRQoL does not sig nificantly improve for patients or their carers following gastrostomy insertion. Given that gastrostomy feeding has no positive effect on HRQoL, questions must be raised as to the merits of this intervention if it only serves to improve physiological outcomes.

Disclosure of Interest None Declared.

\section{PWE-184 THE ASSOCIATION OF DIETICIAN FOLLOW UP WITH THE QUALITY OF LIFE IN COELIAC DISEASE}

doi:10.1136/gutjnl-2013-304907.472

1."M A Zubir, ${ }^{2 B}$ Engel. 'Gastroenterology Department, East Sussex Healthcare NHS Trust, Hastings; ${ }^{2}$ Faculty of Health and Medical Sciences, University of Surrey, Guildford, UK

Introduction Coeliac disease (CD) is present in 1\% of the UK population however there are still many undiagnosed cases in the community. The main treatment for this condition is gluten free diet. The role of dieticians in the follow up care of CD patients is crucial especially with regards to educating CD patients about their dietary requirement as well as their role in disease monitoring. The aim of the study is to assess the association of dieticians follow up on the quality of life in CD patients.

Methods CD patients who were identified on histology over a 10 years period in East Sussex were sent a socio-demographic questionnaire and the Coeliac Disease Quality of Life (CD-OOL) survey by post. The participants of the study who attend dietician follow up were then compared with those who did not. Multiple linear and logistic regression analyses were used to identify any significant relationships

Results Overall, 58 participants (34\%) completed and returned the questionnaires. Dietician involvement appeared to be the most important predictor (beta $=-0.424, \mathrm{p}=0.001$ ) for overall CDOOL score but surprisingly, the negative value indicates that dietician involvement was associated with a lower quality of life score. Increasing age seemed to have a positive impact (beta $=0.312$, $p=0.011$ ) on overall quality of life. However, a change of the recent gluten free prescription (beta $=-0.246, p=0.037$ ) which limits the products available and having initially presented with gastrointestinal symptoms (beta $=-0.24, p=0.044$ ) were found to have negative contributions to the overall CDQOL score. The overall model fit was moderate $\left(R^{2}=0.336\right)$. Logistic regression was used to identify the likelihood of dietician follow up and the only significant predictor identified was the overall CDQOL score (OR 0.95, p = 0.019) which indicates that those with higher quality of life score were less likely to have dietician follow up.

Conclusion Dietician follow up is associated with poorer quality of life but due to the nature of this study, cause and effect cannot be established. It may be that patients who have poorer quality of life chose to see a dietician and longitudinal study is required to further assess this association. This study also suggests that the recent change to the gluten free prescription has negatively impacted the quality of life of those with CD. This fact should be taken into consideration for any future health providers dealing with local CD service provisions.

Disclosure of Interest None Declared.

\section{PWE-185 USE OF DEEP SEDATION FOR A PERCUTANEOUS GASTROPEXY SERVICE IN A DISTRICT GENERAL HOSPITAL}

doi:10.1136/gutjnl-2013-304907.473

1."N Siddique, 'C Nunes, ' A F Muller. 'Gastroenterology, Kent and Canterbury Hospital, Canterbury, UK

Introduction All patients with head and neck cancer referred for nutritional support with enteral feeding to our unit undergo direct 